\title{
Influencia de la formación en competencia cultural sobre la actitud frente a la inmigración de los estudiantes de tercero de enfermería
}

\author{
Rosa Rifà-Ros, Lluís Costa-Tutusaus, Carme Espinosa-Fresnedo
}

Introducción. La formación de las futuras enfermeras plantea la necesidad de preparar profesionales competentes para poder afrontar las incertidumbres y la complejidad que genera la diversidad cultural, entendiendo que cuidar debe contemplar el respeto y la reflexión crítica sobre las ideas y los valores implicados en el dialogo que se genera en la relación terapéutica. El objetivo de este trabajo es evaluar la modificación de la actitud hacia la inmigración en los estudiantes de tercero de enfermería que han cursado la asignatura 'Enfermería y multiculturalidad'.

Sujetos y métodos. Para evaluar la actitud de los estudiantes se ha utilizado la escala de actitud ante la inmigración para enfermería (EAIE) en una muestra apareada formada por los 33 estudiantes de tercer curso de enfermería matriculados en la asignatura optativa 'Enfermería y multiculturalidad', mediante la comparación entre las puntuaciones obtenidas en la escala antes y después de cursarla. Para estimar la significación de las diferencias entre las puntuaciones pre y postintervención se ha utilizado la prueba no paramétrica de Wilcoxon para muestras apareadas, tanto para la puntuación global como para cada uno de los ítems de la EAIE.

Resultados. Con el análisis individualizado de los ítems se observa que, del total de 39 ítems, siete han presentado diferencias significativas $(p<0,05)$ en las mediciones iniciales y finales.

Conclusión. La 'enculturización' enfermera que reciben los estudiantes en su proceso formativo influye favorablemente en la toma de conciencia de sus propias actitudes.

Palabras clave. Actitud. Estudiantes de enfermería. Formación. Inmigración.

The influence of education in cultural competence on third level nursing students' attitudes towards immigration

Introduction. The training of future nurses will have to produce professionals who are competent to face the uncertainties and complexities generated by cultural diversity. Nursing care includes respect and critical reflection on the ideas and values arising from the dialogue generated throughout the therapeutic relationship. The objective of this study was to assess the change of the module 'Nursing and Multiculturalism' in modifying third level nursing students' attitudes towards immigration in the students that were enrolled.

Subjects and methods. To evaluate the attitude of the students we used the Attitude Scale towards immigration for nursing (EAIE). A paired sample was employed, drawn from the 33 third year nursing students enrolled in the optional module 'nursing and multiculturalism' by comparing scores achieved in the scale towards immigration before and after attending the module. In order to estimate the significance of the different rates pre- and post-test, a non-parametric test for paired samples (Wilcoxon signed rank test) was used, both for global rating and for each of the item in the EAIE questionnaire.

Results. The individual analysis of the items shows that the total 39 items, seven presented significant differences $(p<0.05)$ in the initial and final measurements.

Conclusion. Nursing enculturation experienced during basic education, positively influences students in making conscious their attitudes towards immigration.

Key words. Attitude. Education. Immigration. Nursing students.
Facultad de Ciencias de la Salud Blanquerna. Universitat Ramon Llull. Barcelona, España.

Correspondencia:

Dra. Rosa Rifà Ros. Facultad de Ciencias de la Salud Blanquerna. Universitat Ramon Llull. Padilla, 326-332. E-08024 Barcelona.

Fax: + 34932533086 .

E-mail:

rosarr@blanquerna.url.edu

Conflicto de intereses: No declarado.

Competing interests: None declared.

(C) 2013 FEM 


\section{Introducción}

Los últimos datos publicados por la Secretaría de Estado de Inmigración y Emigración del Ministerio de Trabajo y Emigración, a finales de marzo de 2012, apuntan que el número total de residentes extranjeros en situación regularizada en el conjunto del Estado español era de 5.294.720 personas. Respecto a Cataluña, según la misma fuente y en el mismo periodo, el número total era de 1.215 .150 personas, cifra que supone aproximadamente el $23 \%$ del total [1].

La inmigración se considera un fenómeno bilateral que afecta a quien decide embarcarse en un proceso migratorio, pero también a los autóctonos que acogen a estas personas inmigradas [2,3].

Gertz [4] concibe las culturas como mecanismos de control para regir el comportamiento. Según el autor, la cultura tiene unos rasgos fácilmente observables, que llama 'cultura explícita' y que incluye conductas, apariencia, hábitos, lenguaje, indumentaria, costumbres, tradiciones y creencias, y una serie de aspectos que habitualmente no están en nuestra consciencia ni en la de los otros, que corresponderían a la 'cultura implícita'. Ésta incluye normas, percepción, orientación temporal, orientación espacial, estilos de aprendizaje, personalidad, roles, expectativas, valores y actitudes. Según esto, el proceso de 'enculturación' que nos hace miembros de una cultura determinada es el que nos lleva a adoptar ciertas actitudes hacia las otras realidades culturales.

Los diferentes perfiles culturales de las personas a quienes cuidan los profesionales de enfermería crean tensiones y malestar en este colectivo porque se encuentran cuidándolos con pocos conocimientos de los rasgos característicos de su cultura de procedencia. El reto de cuidar en una sociedad multicultural a menudo fracasa porque falla la comunicación a raíz del desconocimiento cultural, las imágenes estereotipadas, las actitudes negativas e incluso el rechazo hacia la diferencia cultural [5].

En España, a partir de los años noventa, se han hecho diversos estudios que han descrito de manera sistemática las actitudes de la población autóctona frente a la inmigración [6,7]. Los indicadores habituales que se miden en estas encuestas no sirven para los profesionales de enfermería porque recogen aspectos demasiado generales y poco vinculados al ámbito de la relación profesional. Es por ello que se deben valorar las actitudes de los profesionales de enfermería con instrumentos que midan actitudes vinculadas a sus relaciones con las personas de otras culturas en un contexto profesional y que reflejen las actitudes que se dan en estas situaciones para poder analizarlas y aplicar medidas para su modificación [8]. En este sentido, se precisa la formación de los profesionales de enfermería en competencia cultural como mecanismo para afrontar y dar una respuesta adecuada a los nuevos retos sociosanitarios que plantea la diversidad cultural en nuestro sistema sanitario $[9,10]$.

Según esto, es importante dirigir también los esfuerzos hacia los futuros profesionales de enfermería para ayudarlos a adquirir una sensibilidad hacia los procesos culturales de las personas, independientemente de su origen, para que puedan reconocer sus propias actitudes, ya sean positivas o negativas, y sepan gestionarlas en su relación con los pacientes $[2,8]$.

Según el modelo de competencia cultural de Purnell [11], el proveedor de cuidados tiene la responsabilidad de ser competente culturalmente. La competencia cultural es conocer, utilizar y apreciar la cultura del otro al colaborar en la resolución de un problema. El proveedor de cuidados necesita poseer un autoconocimiento de su existencia, sensaciones, pensamientos y ambiente sin permitir que estos factores tengan un efecto inadecuado en los destinatarios de sus cuidados. Lo que diferencia el modelo de Purnell de otros enfoques en competencia cultural es el énfasis en la necesidad de que el profesional sea consciente de sí mismo y se autoexplore personalmente. En este sentido considera que ciertas actitudes silenciosas o inconscientes, o la consciencia de las propias creencias, pueden afectar negativamente en el resultado del proceso terapéutico [12].

Algunos estudios investigan sobre la competencia cultural de los profesionales en diferentes ámbitos y en ellos se muestra la insatisfacción de estos profesionales con la calidad de su trabajo, que se relaciona con la inadecuada formación en competencia cultural y con la inexistencia de protocolos y programas en este sentido [13,14].

Formar para el desarrollo de la competencia cultural significa potenciar un conjunto de actitudes y aptitudes integrales que promuevan el respeto y el diálogo, la tolerancia y el sentido critico, y la empatía y la afirmación de la propia identidad para garantizar la comunicación, el diálogo y la escucha activa, rasgos imprescindibles para ofrecer unos cuidados culturalmente competentes.

Como se ha mencionado, esta formación actitudinal de las enfermeras no debe posponerse a la formación posgraduada o a la adquisición de experiencia profesional, sino que debe contemplarse en los planes de estudios del grado en enfermería, marcando unos objetivos que permitan al estudiante aprender los contenidos y desarrollar las habilida- 
des y las actitudes necesarias para la adquisición de la competencia cultural $[2,15]$.

Una de las competencias de los programas del grado en enfermería es conocer la diversidad cultural de nuestro entorno social, analizando los principales problemas socioculturales que se articulan, integrando valores y actitudes positivas hacia la diferencia y adoptando la interculturalidad como forma de convivencia y enriquecimiento personal. La diversidad es constitutiva de cualquier sociedad y le confiere una gran complejidad. Para alcanzar este objetivo, se hace evidente la necesidad de diseñar el aprendizaje desde la lógica del pensamiento y los saberes complejos [16].

En los estudios de enfermería se parte de una constatación evidente, en general, y salvando algunas excepciones, los estudiantes al inicio tienen una información, visión y opinión de la diversidad cultural sesgada y distorsionada por el etnocentrismo propio de la sociedad occidental y por numerosos estereotipos y prejuicios hacia las otras culturas. La teoría y el método enfermero son esenciales para ofrecer cuidados de calidad, pero también lo son las actitudes y valores hacia uno mismo y hacia el otro [17].

Por ello, la hipótesis de esta investigación es que la formación en competencia cultural modifica positivamente la actitud de los estudiantes de enfermería hacia la inmigración y sus objetivos son identificar cuál es la actitud de los estudiantes de tercero de enfermería hacia la inmigración y evaluar la modificación de la actitud hacia la inmigración en los estudiantes de tercero de enfermería que han cursado la asignatura 'Enfermería y multiculturalidad' [18].

\section{Sujetos y métodos}

\section{Ámbito}

Estudiantes de tercer curso de enfermería de la Facultad de Ciencias de la Salud Blanquerna de la Universitat Ramon Llull, en Barcelona.

\section{Diseño y muestra}

Estudio observacional comparativo antes-después. Se ha utilizado una muestra apareada formada por 33 estudiantes matriculados en la asignatura optativa 'Enfermería y multiculturalidad'.

\section{Variables de estudio}

Se han recogido variables sociodemográficas (sexo y edad) y la variable 'actitud ante la inmigración', me-
Tabla I. Escala de actitud hacia la inmigración para enfermería.

1. El extranjero que delinque debería ser expulsado del país ${ }^{b}$

2. La llegada de inmigrantes ha favorecido el aumento de la delincuencia ${ }^{b}$

3. Debemos garantizar que los inmigrantes puedan mantener sus costumbres y pautas culturales ${ }^{a}$

4. La diversidad étnica enriquece la cultura de nuestro país ${ }^{a}$

5. Debemos hacer un mayor esfuerzo para proporcionar a los inmigrantes una mayor calidad de vida a

6. Los inmigrantes, legales e ilegales, deben tener acceso a la sanidad y la educación de forma libre y gratuita ${ }^{a}$

7. Hay colectivos de inmigrantes incapaces de integrarse en nuestra sociedad ${ }^{b}$

8. La diversidad de culturas conlleva cierta pérdida de nuestros valores ${ }^{b}$

9. En ocasiones, los inmigrantes reciben mayores ayudas sociales que los propios autóctonos ${ }^{b}$

10. Los inmigrantes, en general, son poco cuidadosos con su higiene personal ${ }^{b}$

11. Muchos inmigrantes no son respetuosos con los derechos humanos

12. Muchos inmigrantes se aprovechan de los recursos sanitarios y sociales conseguidos con nuestro esfuerzo de muchos años ${ }^{b}$

13. Estamos obligados a respetar la cultura y las costumbres de los inmigrantes ${ }^{b}$

14. Es necesario hacer un esfuerzo para conseguir que los inmigrantes se incorporen a nuestra sociedad como ciudadanos de pleno derecho ${ }^{\text {a }}$

15. En general, los inmigrantes hacen muy poco por integrarse en nuestra sociedad ${ }^{b}$

16. Los inmigrantes ocupan puestos de trabajo que dejan en el paro a muchos de nosotros ${ }^{b}$

17. Los inmigrantes tienen la obligación de adaptarse a las costumbres de nuestro país ${ }^{b}$

18. Debemos favorecer que los inmigrantes puedan disponer de lugares de culto donde practicar sus creencias y religiones ${ }^{\text {a }}$

19. La convivencia de diversas culturas favorece el conocimiento y el mutuo respeto ${ }^{a}$

20. La inmigración y el consiguiente aumento de la natalidad favorece a los países con dificultades para mantener el equilibrio demográfico ${ }^{a}$

21. Los inmigrantes ilegales no deberían tener acceso a la salud pública ${ }^{b}$

22. En ocasiones, pueden justificarse ciertos actos violentos sobre los inmigrantes ${ }^{b}$

23. En gran medida, el colapso de la sanidad pública está provocado por el aumento de la inmigración ${ }^{b}$

24. Se dedican demasiados recursos para la atención a los inmigrantes ${ }^{b}$

25. Un ciudadano europeo afincado en España reporta más beneficios para nuestra sociedad que un inmigrante africano, asiático o sudamericano ${ }^{\text {b }}$

26. Los inmigrantes y los gitanos dificultan el desarrollo del país ${ }^{b}$

27. Los inmigrantes que no se integran es porque no quieren ${ }^{b}$

28. Los hijos de los inmigrantes, legales o ilegales, tienen derecho a la escolarización gratuita a

29. Todos los inmigrantes deben disfrutar de las mismas condiciones de trabajo que los naturales del país a

30. Si seguimos así, a medio plazo, las costumbre y creencias de los inmigrantes se impondrán en nuestra sociedad ${ }^{b}$

31. Las minorías étnicas constituyen una fuente de conflicto social ${ }^{b}$

32. Todas las personas que viven en un mismo país, independientemente de su procedencia o de su etnia, deben tener los mismos derechos ${ }^{\text {a }}$

33. En realidad, nos aprovechamos de los inmigrantes para ofrecerles los trabajos más precarios a

34. A medio plazo, la llegada masiva de inmigrantes provocará graves problemas sanitarios y sociales ${ }^{\text {a }}$ 35. Si puedo elegir, prefiero no sentarme al lado de un inmigrante en un transporte público ${ }^{a}$

36. En general, no soporto a los magrebíes ${ }^{b}$

37. Temo por mi seguridad cuando veo un grupo de magrebíes ${ }^{b}$

38. El Estado debería dedicar más recursos a la integración de las minorías a

39. Es injusto asociar el fenómeno de la inmigración con el aumento de la delincuencia a

a Puntuación: de 1 (total desacuerdo) a 4 (total acuerdo); ' P Puntuación: de 4 (total desacuerdo) a 1 (total acuerdo) 
Tabla II. Resumen descriptivo de las puntuaciones totales antes y después con $Z$ de Wilcoxon y significación (p).

\begin{tabular}{|c|c|c|c|c|c|c|}
\hline & Media & $\begin{array}{c}\text { Desviación } \\
\text { estándar }\end{array}$ & Mediana & p25-p75 & $Z$ de Wilcoxon & $p$ \\
\hline Total inicial & 107,82 & 14,055 & 114,00 & $96-116$ & \multirow{2}{*}{$-0,388$} & \multirow{2}{*}{0,698} \\
\hline Total final & 109,94 & 18,542 & 111,00 & $98-124$ & & \\
\hline
\end{tabular}

Tabla III. Resumen de los ítems modificados significativamente.

\begin{tabular}{lcc}
\hline & $p$ & Sentido del rango \\
\hline $\begin{array}{l}\text { 2. La llegada de inmigrantes ha favorecido } \\
\text { el aumento de la delincuencia }\end{array}$ & 0,029 & $\begin{array}{c}\text { Creciente } \\
\text { (tendencia a total desacuerdo) }\end{array}$ \\
\hline $\begin{array}{l}\text { 4. La diversidad étnica enriquece } \\
\text { la cultura de nuestro país }\end{array}$ & 0,048 & $\begin{array}{c}\text { Decreciente } \\
\text { (tendencia a total desacuerdo) }\end{array}$ \\
\hline $\begin{array}{l}\text { 8. La diversidad de culturas conlleva } \\
\text { cierta pérdida de nuestros valores }\end{array}$ & 0,029 & $\begin{array}{c}\text { Creciente } \\
\text { (tendencia a total desacuerdo) }\end{array}$ \\
$\begin{array}{l}\text { 16. Los inmigrantes ocupan puestos de trabajo } \\
\text { que dejan en el paro a muchos de nosotros }\end{array}$ & 0,01 & $\begin{array}{c}\text { Decreciente } \\
\text { (tendencia a total acuerdo) }\end{array}$ \\
\hline $\begin{array}{l}\text { 17. Los inmigrantes tienen la obligación de } \\
\text { adaptarse a las costumbres de nuestro país }\end{array}$ & 0,029 & $\begin{array}{c}\text { Creciente } \\
\text { (tendencia a total desacuerdo) }\end{array}$ \\
\hline $\begin{array}{l}\text { 26. Los inmigrantes y los gitanos } \\
\text { dificultan el desarrollo del país }\end{array}$ & 0,029 & $\begin{array}{c}\text { Creciente } \\
\text { (tendencia a total desacuerdo) }\end{array}$ \\
\hline $\begin{array}{l}\text { 39. Es injusto asociar el fenómeno de la } \\
\text { inmigración con el aumento de la delincuencia }\end{array}$ & 0,017 & $\begin{array}{c}\text { Creciente } \\
\text { (tendencia a total acuerdo) }\end{array}$ \\
\hline
\end{tabular}

dida con la escala de actitud ante la inmigración para enfermería (EAIE).

\section{Instrumento}

La EAIE es un cuestionario autoadministrado que consta de 39 ítems con formato de respuesta tipo Likert, con cuatro respuestas cada una de ellas. Estas opciones van desde el 'total acuerdo' hasta el 'total desacuerdo', con una puntuación de 1 a 4 o a la inversa, en función de la direccionalidad del reactivo propuesto. Los dos extremos de puntuación están constituidos por una puntuación máxima de 156, que indica las actitudes más favorables hacia la inmigración, y una puntuación mínima de 39, que indica las actitudes más negativas y prejuiciosas.

La EAIE fue desarrollada y validada por Antonin y Tomás-Sábado [8]. En las pruebas de validación de la escala se obtuvo un coeficiente $\alpha$ de Cronbach de 0,94 , un coeficiente test-retest de 0,90 y una es- tructura factorial que apoya su consideración como medida básicamente unifactorial (Tabla I).

\section{Procedimiento}

El estudio se realizó durante el primer cuatrimestre del curso 2010-2011 en la Facultad de Ciencias de la Salud Blanquerna de la Universitat Ramon Llull.

Los cuestionarios se administraron colectivamente a los estudiantes por la profesora de la asignatura el primer y el último día, es decir, antes y después de cursar la asignatura. Previamente se les advirtió que la participación era voluntaria y se garantizó la confidencialidad. Para poder realizar la comparación a cada estudiante se adjudicó el mismo código en ambas mediciones.

\section{Análisis de datos}

Para estimar la significación de las diferencias entre las puntuaciones pre y post se ha utilizado la prueba no paramétrica de Wilcoxon para muestras apareadas, tanto para la puntuación global como para cada uno de los ítems de la EAIE. El nivel de significación para todo el estudio ha sido de 0,05. Los datos se han tabulado y analizado mediante el programa estadístico SPSS v. 19.0.

\section{Resultados}

De los 33 estudiantes matriculados, 19 respondieron a la escala en septiembre del 2010, 25 en diciembre de 2010 y sólo 18 respondieron en ambas ocasiones, por los que son los que podremos comparar. De estos 18 estudiantes, sólo dos son varones, por lo que no utilizaremos la variable sexo como factor a analizar. La edad media de los participantes es de 22 años (rango: 20-45 años).

La tabla II contiene el resumen de las puntuaciones del grupo antes y después de la formación, así como los valores $Z$ de Wilcoxon y su significación para la puntuación global del test. Se observa que no existen diferencias significativas en la actitud de los estudiantes hacia la inmigración antes y después de cursar la asignatura, si bien cabe destacar que ya partíamos de una puntuación media muy alta de 108 (rango: 88-130 puntos).

Para poder realizar un análisis pormenorizado de las diferencias pre y postintervención se comparan las puntuaciones individuales de cada ítem en los dos momentos. Los ítems que han presentado diferencias significativas $(p<0,05)$ en las mediciones iniciales y finales son siete $(2,4,8,16,17,26$ y 39). 
La tabla III resume estas diferencias identificadas, así como el sentido del cambio producido.

A continuación realizamos un análisis individual de cada ítem en los que se han hallado diferencias significativas tomando en consideración el sentido del rango de dicha diferencia:

- Los ítems 2, 8, 17 y 26, que afirmaban que 'la llegada de inmigrantes ha favorecido el aumento de la delincuencia', que 'la diversidad de culturas conlleva cierta pérdida de nuestros valores', que 'los inmigrantes tienen la obligación de adaptarse a las costumbres de nuestro país' y que 'los inmigrantes y los gitanos dificultan el desarrollo del país', respectivamente, presentan una diferencia significativa con sentido creciente y tendiendo al total desacuerdo, por lo que observamos que los estudiantes en relación a estos ítems tienen, después de la realización de la asignatura, una actitud más adecuada desde la perspectiva de la competencia cultural.

- El ítem 39, que afirmaba que 'es injusto asociar el fenómeno de la inmigración con el aumento de la delincuencia, presenta una diferencia significativa con sentido creciente y tendiendo al total acuerdo, lo cual de nuevo evidencia un cambio de actitud hacia una mayor competencia cultural al finalizar la asignatura.

- El ítem 4, que afirmaba que 'la diversidad étnica enriquece la cultura de nuestro país', presenta una diferencia significativa con sentido decreciente y tendiendo al total desacuerdo.

- El ítem 16, que afirmaba que 'los inmigrantes ocupan puestos de trabajo que dejan en el paro a muchos de nosotros', presenta una diferencia significativa con sentido creciente y tendiendo al total acuerdo.

\section{Discusión}

En los resultados generales obtenidos se evidencia que no existen diferencias significativas en la actitud de los estudiantes hacia la inmigración antes y después de cursar la asignatura, si bien cabe destacar que ya partíamos de una puntuación promedio muy alta de 108 . Teniendo en cuenta que esta asignatura se imparte en el tercer año de la titulación podemos pensar que, de acuerdo con los resultados de Antonin y Tomás-Sábado [10], ha tenido una gran influencia el énfasis docente en el principio de equidad hacia todos los usuarios, sean o no de culturas diversas.

Con el análisis individual de los ítems descritos en el apartado anterior podemos realizar matices en la afirmación de que no existen diferencias entre las actitudes pre y postintervención, puesto que el resultado de los ítems 2, 8, 16, 27 y 39 denota que los estudiantes, después de cursar la asignatura, muestran una actitud más adecuada desde la perspectiva de la competencia cultural, y el resultado de los ítems 4 y 6 , un retroceso respecto a la actitud ante la inmigración en relación a la puntuación postintervención.

En los resultados de estos ítems se observa una clara contradicción entre el ítem 4, en el que se afirma que 'la diversidad étnica enriquece la cultura de nuestro país', con el que los estudiantes se muestran de acuerdo, y el ítem 8, que afirma que 'la diversidad de culturas conlleva cierta pérdida de nuestros valores', con el que los estudiantes se mostraban en claro desacuerdo.

En relación a estos resultados cabe destacar que el momento del curso en el que se realizó la asignatura y las dos mediciones coincidió con la campaña electoral de las elecciones autonómicas de Cataluña, lo que hace pensar en la posible influencia negativa de los medios de comunicación, que resaltaban las campañas de algunos de los candidatos a dichas elecciones, muy contrarias y represivas en relación al fenómeno migratorio.

En el mismo sentido, el ítem 16, que afirmaba que 'los inmigrantes ocupan puestos de trabajo que dejan en el paro a muchos de nosotros', presenta una diferencia significativa con sentido creciente y tendiendo al total acuerdo. El resultado obtenido en este ítem plantea hasta qué punto el actual momento de crisis que estamos viviendo mundialmente no está teniendo una influencia mayor que el que pueda ejercer la asignatura en una afirmación con un claro componente económico. Algunos estudios $[19,20]$ destacan la importancia de los medios de comunicación en la creación y transmisión de unos estereotipos muy definidos, en referencia a la imagen del inmigrante.

Con estos resultados cabe pensar que la 'enculturización' enfermera que reciben los estudiantes en su proceso formativo influye favorablemente en la toma de conciencia de sus propias actitudes, lo cual, siguiendo la propuesta del modelo de competencia cultural de Purnell $[11,12]$, les permite desarrollar una mayor sensibilidad hacia el respeto y la necesidad de contemplar el bagaje cultural de las personas, así como adquirir un mayor compromiso con la diferencia cultural.

De acuerdo con Spector [21,22] son imprescindibles los cuidados culturales para ofrecer respuestas altamente complejas a las personas de otras culturas. 'Cuidados culturales' es un concepto que describe que los cuidados enfermeros deben ser cultu- 
ralmente sensibles, culturalmente congruentes y culturalmente competentes.

En conclusión, las características del estudio nos hacen ser prudentes en conferir a la asignatura el peso total del cambio de actitud de los estudiantes, puesto que en su diseño no contemplamos la existencia de un grupo control. No obstante, dado que el periodo entre mediciones es breve -se han realizado con cuatro meses de diferencia-, tampoco podemos plantear que el cambio se deba a un proceso natural de maduración, por lo que de nuevo debemos considerar la influencia ejercida por la asignatura.

Aunque los resultados de nuestro estudio permiten un cierto grado de optimismo en cuanto a la modulación de las actitudes individuales por parte de nuestros estudiantes, debemos seguir trabajando en este sentido porque el contexto sociopolítico y económico ejerce también un gran impacto en la construcción de sus actitudes y, por lo tanto, en la adquisición de la competencia cultural requerida.

\section{Bibliografía}

1. Ministerio de Trabajo y Emigración. Secretaria de Estado de Inmigración y Emigración. URL: http://extranjeros.empleo. gob.es/es/Estadisticas/operaciones/con-certificado/201203/ Principales_resultados_31032012.pdf. [28.08.2012].

2. Moreno-Preciado M, Agudo-Gisbert E. Formación y transculturalidad: el cuidado del paciente inmigrado desde la perspectiva del estudiante de enfermería de primer año. Cultura de los Cuidados 2006; 20: 55-61.

3. O'Mahony JM, Donelly TT. The influence of culture on immigrant women's mental health care experiences from the perspectives of health care providers. Issues Ment Health Nurs 2007; 28: 453-71.

4. Gertz C. La interpretación de las culturas. Barcelona: Gedisa; 2001.

5. Plaza del Pino FJ, Martínez-Gálvez L, Rodríguez-Alonso J, Plaza del Pino MD. Visión de los profesionales de enfermería sobre los pacientes marroquíes. Metas de Enfermería 2007-2008; 10: 27-30.
6. Cea d'Ancona MA. La medición de las actitudes ante la inmigración: evaluación de los indicadores tradicionales de racismo. Rev Reis. 99/02; 87-111.

7. Navas MS, Cuadrado I. Actitudes hacia gitanos e inmigrantes africanos: estudio comparativo. Apuntes de Psicología 2003; 21: 29-49.

8. Antonín-Martín M, Tomás-Sábado J. La escala de actitud ante la inmigración para enfermería: validación preliminar Enfermería Científica 2004; 262-263: 77-82.

9. Ibarra-Mendoza TX, Siles-González J. Competencia cultural: una forma humanizada de ofrecer cuidados de enfermería. Índex de Enfermería (Gran) 2006; 55: 44-8.

10. Antonín-Martín M, Tomás-Sábado J. Actitud ante la inmigración en estudiantes de enfermería. Valoración antes y después del 11-M. Educ Med 2006; 9: 17-20.

11. Purnell $\mathrm{L}$. The Purnell model for cultural competence. J Transcult Nurs 2002; 13: 193-6.

12. Purnell L. A description of the Purnell model for cultural competence. J Transcult Nurs 2000; 11: 40-6.

13. Berlin A, Johansson SE, Törnkvist L. Working conditions and cultural competence when interacting with children and parents of foreign origin: Primary Child Health Nurses' opinions. Scand J Caring Sci 2006; 20: 160-8

14. Van den Brink Y. Diversity in care values and expressions among Turkish family caregivers and Dutch community nurses in The Netherlands. Transcult Nurs 2003; 14: 146-54.

15. Fernández-Donaire L, Fernández-Narváez $\mathrm{P}$, Tomás-Sábado $\mathrm{T}$. Modificación de actitudes ante el sida en estudiantes de enfermería: resultados de una experiencia pedagógica. Educ Med 2006; 9: 84-90.

16. Morera-Pomerade MJ, Roca-Caparà N. El aprendizaje reflexivo de la diversidad cultural. Rev Rol Enf 2007; 30: 699-704.

17. Leno-González D, Leno-González JL, Lozano-Guerrero MJ. Aspectos socioculturales con influencia en la salud y la enfermedad: repercusión de los cuidados enfermeros. Metas de Enfermería 2007; 10: 58-64.

18. Pallarés-Martí A, Rifà-Ros R. Enfermería y multiculturalidad: estrategia docente para afrontar la diversidad. Metas de Enfermería 2005; 8: 16-9.

19. Van Gorp B. Where is the frame? Victims and intruders in the Belgian press coverage of the asylum issue. Eur J Commun 2005; 20: 484-507.

20. Igartua JJ, Muñiz C. Encuadres noticiosos e inmigración. Un análisis de contenido de la prensa y televisión españolas. Zer. Revista de Estudios de Comunicación 2004; 16: 87-104.

21. Spector RE. Cultural diversity in health and illness. 6 ed. Upper Saddle River: Prentice Hall Health; 2004.

22. Spector RE. Valoración de la herencia cultural. Cultura de los Cuidados 2001; 9: 74-81. 